\title{
In Vivo Evaluation of a Decellularized Limbal Graft for Limbal Reconstruction
}

\author{
Maryam A. Shafiq, Behrad Y. Milani, and Ali R. Djalilian \\ Department of Ophthalmology and Visual Sciences, University of Illinois at Chicago, Chicago, IL 60612, USA \\ Correspondence should be addressed to Ali R. Djalilian; adjalili@uic.edu
}

Received 30 June 2013; Revised 15 October 2013; Accepted 7 January 2014; Published 12 February 2014

Academic Editor: Raymund E. Horch

Copyright (C) 2014 Maryam A. Shafiq et al. This is an open access article distributed under the Creative Commons Attribution License, which permits unrestricted use, distribution, and reproduction in any medium, provided the original work is properly cited.

\begin{abstract}
Corneal and limbal epithelial function is highly dependent on its underlying matrix. In this study, we report the in vitro and in vivo effects of a decellularized limbal matrix on corneal and limbal epithelial differentiation and repair. We demonstrate that a limbal matrix helps to maintain epithelial cells in a more proliferative and less differentiated state. We introduce a novel focal injury model to the limbus using an excimer laser and further show that transplanting a decellularized limbal graft after the limbal injury helps to promote epithelialization and reduce corneal haze formation. These results suggest that a decellularized limbal graft may be therapeutically beneficial in clinical cases of focal limbal deficiency.
\end{abstract}

\section{Introduction}

The corneal epithelium forms a barrier that is critical to the integrity and clarity of the cornea. The absence of the epithelium or a compromise in its function renders the cornea susceptible to infections, secondary loss of optical clarity, and ultimately loss of vision. The corneal epithelium is maintained by a reservoir of stem and progenitor cells that are located in the limbal region-the junction between the cornea and the sclera [1-4]. Damage to the limbus can lead to the loss of the stem cells which in turn leads to conjunctival epithelial growth over the cornea-a condition known as limbal stem cell deficiency $[5,6]$.

A number of tissue engineering approaches for the reconstruction of the corneal and limbal epithelium have been investigated [7-12]. In most clinical applications, limbal epithelial cells are expanded in vitro and then transplanted to the diseased corneal surface $[13,14]$. Various substrates for culturing corneal epithelial cells have been reported [15-17]. Currently human amniotic membrane is the most common substrate and carrier for ex vivo cultured human corneal epithelial cells $[18,19]$. Previously, we reported the use of a decellularized cornea as a matrix for expanding corneal cells in vitro [20]. We demonstrated that decellularized human corneas provide a scaffold that can support the growth of corneal epithelial cells and stromal fibroblasts.
In this study, we extend our previous experiments by first showing that cells grown over a decellularized limbal matrix maintain a more limbal phenotype when compared to cells cultured over the central cornea. Next, we use a novel laser based limbal injury model to show that decellularized limbal grafts promote epithelial cell regrowth in vivo and reduce corneal scarring and neovascularization.

\section{Materials and Methods}

2.1. Cell Culture. Human corneal epithelial cultures were initiated from fresh cadaver corneas kindly provided by the Illinois Eye Bank [21]. Briefly, the corneas were rinsed in phosphate buffered saline (PBS) and then incubated in $2 \mathrm{mg} / \mathrm{mL}$ of Dispase II (Invitrogen) in PBS for $1 \mathrm{~h}$ at $37^{\circ} \mathrm{C}$. The epithelial sheets were peeled off and digested in $0.25 \%$ trypsin-EDTA at $37^{\circ} \mathrm{C}$ for $30 \mathrm{~min}$. Cells were washed and then resuspended in keratinocyte serum-free medium (KSFM; Invitrogen), DMEM plus Ham's F12, and fibroblast conditioned media in a ratio of $1: 1: 1$. The cells were then plated on the basement membrane side of the decellularized cornea at a density of $1 \times 10^{6}$ cells/cornea.

2.2. Fibroblast Conditioned Media (CM). After isolating epithelial cells (as described above), the remaining stroma was cut into $1 \times 1 \mathrm{~mm}$ pieces and incubated in $0.1 \%$ 


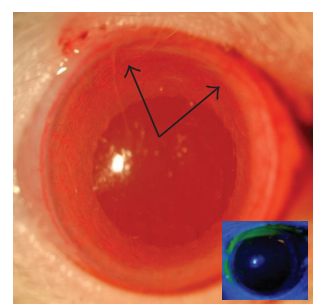

(a)

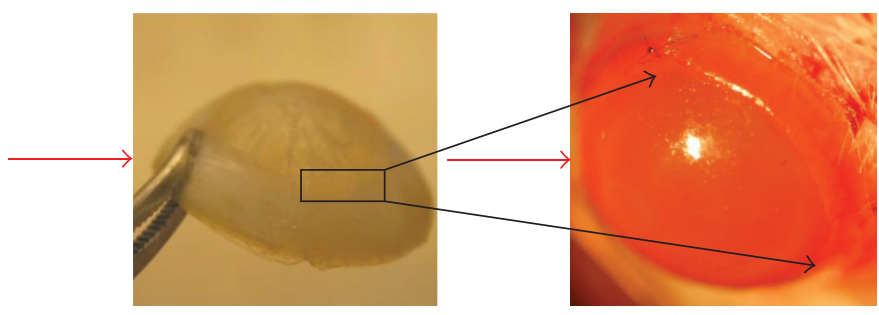

(b)

(c)

FIGURE 1: Experiment was designed to create a focal limbal injury by laser followed by grafting with a decellularized human limbus. (a) Nidek EC-5000 excimer laser system was used to ablate corneal tissue under anesthesia from a rat eye at the limbal area (junction of cornea and sclera). Arrows pointing to the area of ablation. Inset shows ablated area stained with fluorescein. (b) Decellularized human cornea. (c) Decellularized human cornea grafted on the ablated rat eye. Arrows pointing to the graft.

collagenase (Sigma) in DMEM at $37^{\circ} \mathrm{C}$ for $1.5 \mathrm{~h}$ on a shaker. The isolated keratocytes were expanded and differentiated into fibroblasts by culturing in DMEM supplemented with $10 \%$ fetal bovine serum (FBS), $100 \mathrm{U} / \mathrm{mL}$ penicillin, and $100 \mathrm{mg} / \mathrm{mL}$ streptomycin. After 1-2 passages, when the cells had reached at least $80 \%-90 \%$ confluence, the media were changed to DMEM without serum. Twenty-four hours later the media were collected and used immediately. This fibroblasts conditioned media (CM) was mixed in keratinocyte serum-free medium (KSFM; Invitrogen), plus DMEM + Ham's F12 in equal ratio.

2.3. Decellularization of Human Cornea. Corneas were subjected to a stepwise treatment with nonsurfactant treatment involving incubation in $1.5 \mathrm{M}$ sodium chloride $(\mathrm{NaCl})$ solution for $48 \mathrm{~h}$ with $\mathrm{NaCl}$ change after $24 \mathrm{~h}$ in the first step. In the second step, corneas were treated with DNAse $5 \mathrm{U} / \mathrm{mL}$ and RNAse $5 \mathrm{U} / \mathrm{mL}$ (Sigma-Aldrich) for $48 \mathrm{~h}$. Corneas were then washed with PBS for $72 \mathrm{~h}$ with PBS changed every $24 \mathrm{~h}$. The decellularization procedure was carried out at room temperature under continuous agitation.

2.4. Laser Induced Limbal Injury Model. A novel laser ablation technique was used to create reproducible focal injuries to the limbal epithelium in vivo. This technique was developed to have precise control over the size and depth of destroyed epithelium and underlying stroma. This was done using a Nidek EC-5000 excimer laser system in adult Lewis rats. The rats were placed under anesthesia using an injection of ketamine hydrochloride (100 mg/kg IP) and xylazine ( $5 \mathrm{mg} / \mathrm{kg}$ IP) hydrochloride intraperitoneally. For local anesthesia, one drop of proparacaine hydrochloride $0.5 \%$ was applied to the eye. A $1.5 \mathrm{~mm}$ spot size was used to ablate a limbal area of $5.0 \times 1.5 \mathrm{~mm}^{2}$ in size and $100 \mu \mathrm{m}$ in depth.

As a comparison to the laser ablation model, alkali injury and mechanical epithelium debridement injury models were also studied in a mouse's eyes (data not shown). Mechanical debridement was performed using a $23 \mathrm{G}$ needle to remove the epithelium in a focal area. Alkali injury was done using sodium hydroxide $(\mathrm{NaOH})$ with concentrations ranging from $0.25 \mathrm{M}$ to $1 \mathrm{M}$ applied to the eye for 30 seconds.

2.5. Transplantation of a Decellularized Limbal Graft in a Rat Eye. Lamellar transplantation of decellularized limbal grafts was performed on rat eyes following laser injury (Figure 1). The process of preparing the graft for transplantation involved a lamellar dissection to separate the anterior half of the limbus and discarding the posterior stroma, Descemet's membrane, and the endothelial cells. This decellularized limbal graft was secured to the lasered area of the rat eye using interrupted 10-0 nylon sutures. Immediately after the procedure, antibiotic ointment (Bacitracin) was applied to the eye. The antibiotics were given once a day for 7 days. In control rats, only laser injuries were performed without transplantation of a graft. After one week, the rats were euthanized and the eyes were enucleated and subjected to immunohistologic evaluation.

2.6. Slit-Lamp Imaging. The clinical status of the ocular surface in rats was assessed on days 0,3 , and 7 after transplantation. Using a slit-lamp microscope, the degree of opacity, inflammation, and neovascularization was monitored. Epithelialization was assessed by fluorescein staining of the cornea.

2.7. Immunohistologic Evaluation. The corneas were embedded in OCT then cut into $10 \mu \mathrm{m}$ sections. The tissue sections were briefly fixed in $4 \%$ paraformaldehyde for $20 \mathrm{~min}$ followed by permeabilization in $0.3 \%$ Triton-X in tris buffered saline (TBS) for $10 \mathrm{~min}$ at room temperature. The sections were blocked with $10 \%$ donkey serum with $1 \%$ bovine serum albumin (BSA) in TBS for $1 \mathrm{~h}$ at room temperature. The primary antibodies used were mouse anti-p63 (1:100, Santa Cruz Biotechnology), rabbit anti-Ki67 (1:50 Epitomics) or goat anti-keratin 12 antibody (1:50, Santa Cruz). The primary antibodies were incubated either overnight at $4^{\circ} \mathrm{C}$ or for $2 \mathrm{~h}$ at room temperature.

A fluorescein isothiocyanate (FITC) conjugated donkey anti-rabbit or anti-mouse or rhodamine conjugated donkey anti-goat (all 1:300-400, Jackson ImmunoResearch) was applied for $1 \mathrm{~h}$ at room temperature in the dark. For negative control, the primary antibody incubation step was omitted or instead an isotype control was used. Slides were coverslipped with mounting medium with or without DAPI and images were visualized using a Zeiss Axiovert fluorescence microscope and photographed with an AxioCam (Carl Zeiss, Thornwood, NY) camera. 


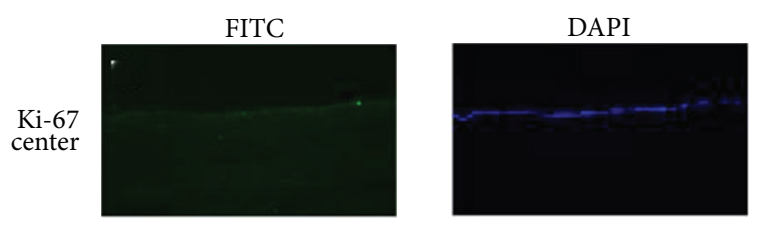

(a)
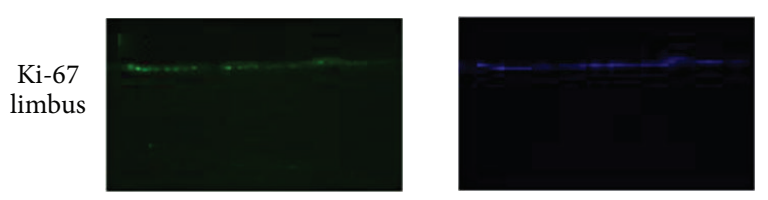

(b)
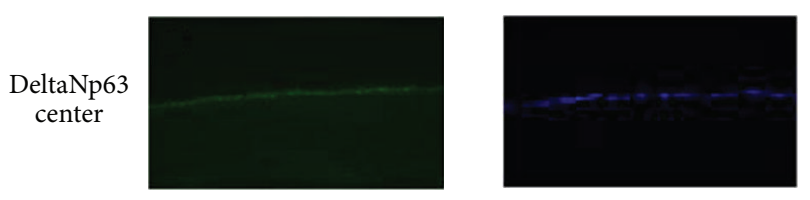

(c)
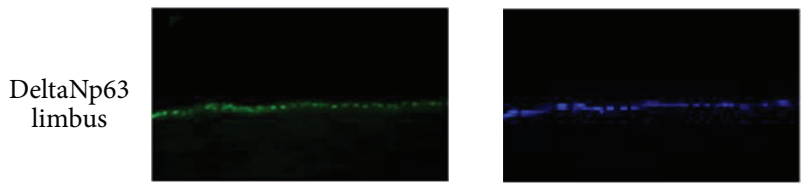

(d)

Figure 2: Corneal epithelial cells grown on decellularized cornea show higher expression of Ki-67, a marker for proliferation, at the limbus as compared to the central cornea ((a) and (b)) and higher expression of limbal stem cell marker DeltaNp63 at the limbus as compared to the central cornea ((c) and (d)).

\section{Results}

3.1. In Vitro Evaluation of Epithelial Cells on Decellularized Cornea and Limbus. In a previous study, we showed that our decellularization method maintained the integrity of the extracellular matrix and basement membrane in the limbal area [20]. In this study, we evaluated the cellular phenotype of the epithelial cells cultured over the limbal and central corneal. The epithelial cells grown on the decellularized limbus presented with a higher degree of proliferation compared to those in the central cornea (Figure 2). Likewise, a higher percentage of cells expressed the limbal stem cell marker DeltaNp63 in the limbal versus central matrix. The results of this experiment show that the decellularized limbal matrix helps support a limbal (i.e., less differentiated and more proliferative) phenotype in the epithelial cells.

3.2. Excimer Laser Focal Limbal Injury Model. We initially tested mechanical and chemical (alkali) measures to create focal limbal injuries. Mechanical debridement was found to only remove the epithelium with minimal injury to the underlying stroma. On the other hand, alkali injury resulted in extensive damage to the surrounding tissue leading to significant inflammation and corneal opacification (data not shown). The extent of limbal injury was highly variable with the alkali injury. The excimer laser, however, created consistent and reproducible levels of epithelial and stromal damage. Upon examination with slit-lamp microscope, a positive fluorescein staining in cornea after laser injury confirmed the absence of the epithelium. Also, upon gross inspection, the laser epithelium removal did not induce excessive inflammation in the cornea (Figure 3). The laser injury method was chosen to study the decellularized limbal grafts.

3.3. In Vivo Evaluation of Decellularized Limbal Grafts after Focal Limbal Injury. We studied the in vivo effects of decellularized limbal tissue in the rat eye following laser ablation of the limbus. The eyes were followed for a period of one week (Figure 3). While both grafted and nongrafted control eyes completely epithelialized within 3 days, the eyes that received grafts healed faster with minimal fluorescein staining and stromal haze. In contrast, the eyes without a graft demonstrated late staining with fluorescein (indicating a compromised epithelial barrier) and greater stromal haze (Figure 4). In addition, the non-grafted eyes demonstrated greater degree of peripheral neovascularization compared to the eyes that received a decellularized limbal graft (Figure 5).

The eyes were removed and sectioned for histological analysis. The grafts were found to have stratified epithelium growing on top with the presence of inflammatory cells in the stroma. Epithelial cells growing on the graft displayed positive staining for keratin 12, a marker for corneal epithelial differentiation (Figure 4).

A total of 8 grafts were performed. In successful grafts, we observed epithelialization of the graft in one week. But in the earlier cases, where thicker grafts had been used, we observed ingrowth of host corneal epithelial cells into the host-graft junction. The host cornea became opaque and neovascularization occurred, resulting in separation and extrusion of the graft.

\section{Discussion}

In this study, we have first shown that a decellularized limbal matrix can maintain a more limbal phenotype in vitro. Specifically, the corneal epithelial cells grown on the decellularized limbus had higher expression of the proliferation maker Ki67 and stem cell marker DeltaNp63 compared to those in the central cornea. These results highlight the critical role of the basement membrane in maintaining the limbal stem cell/progenitor cells. Previous studies have similarly demonstrated the importance of the limbal microenvironment in the limbal stem cell function [22].

We then proceeded with in vivo experiments to test the effect of decellularized limbal constructs. We devised a novel laser ablation method to create well defined and reproducible limbal injuries. Chemical injuries have traditionally been used to create limbal injuries and limbal stem cell deficiency $[23,24]$. However, in our model we wanted to specifically create only focal injury and $\mathrm{NaOH}$ was found to cause excessive and variable degrees of stromal destruction. The main advantage of using the excimer laser is the opportunity to control the ablation depth to remove the epithelium and the immediate underlying matrix. 

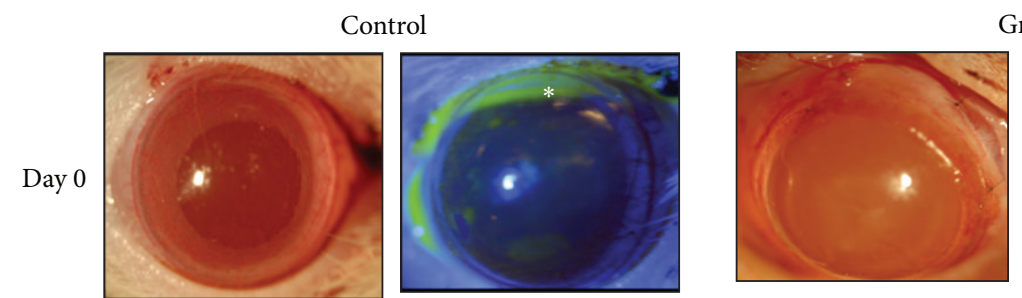

Graft
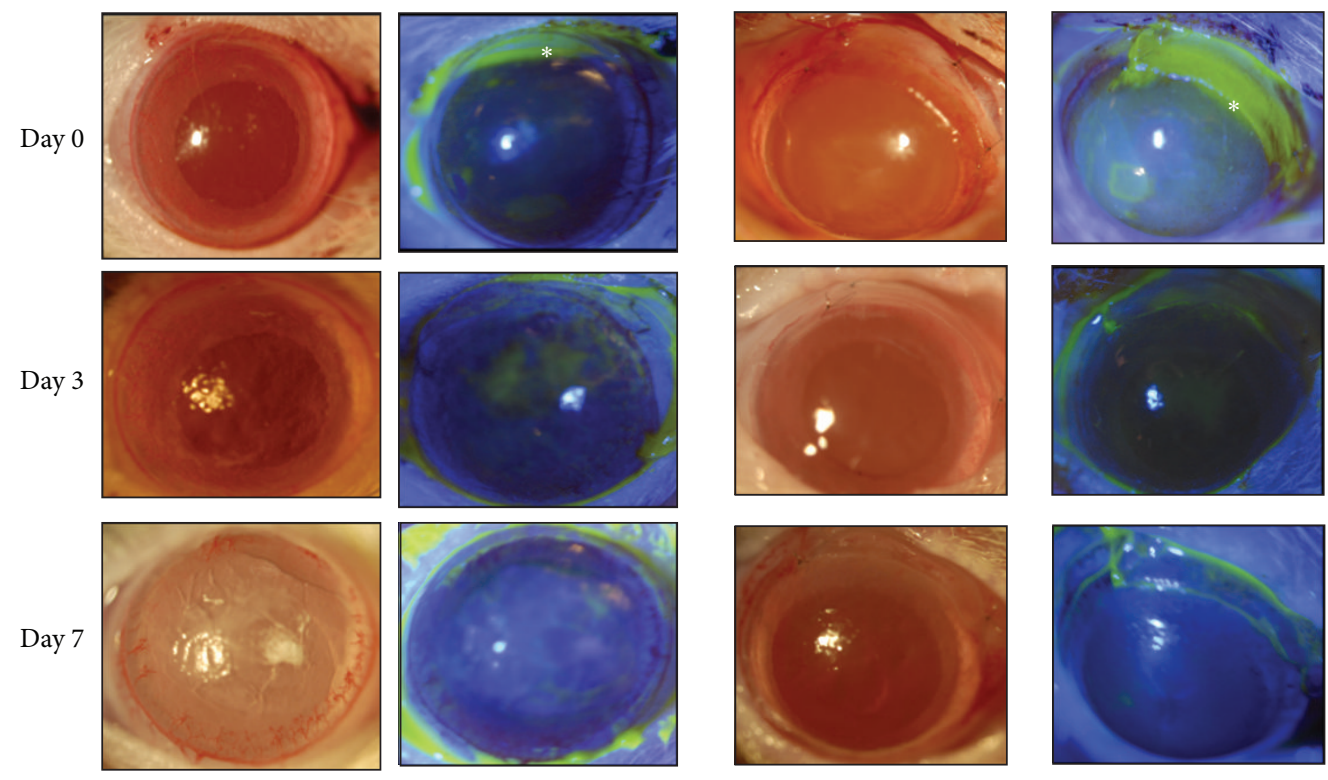

Figure 3: Slit-lamp images with and without fluorescein to evaluate integrity of the graft in the rat eye. Fluorescein detects the absence of epithelium, and damage to the epithelium is represented by positive fluorescein staining. Images were taken at days 0,3 , and 7 . At day 7 , the treated area was completely epithelialized in both control and grafted corneas.

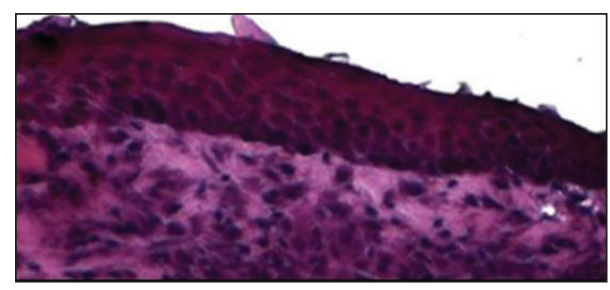

(a)

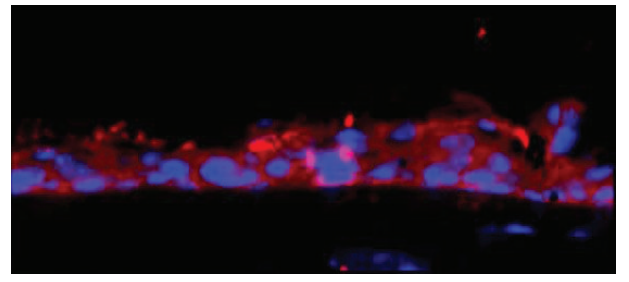

(b)

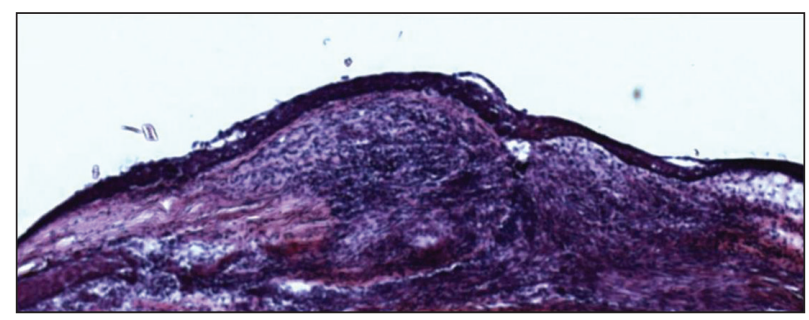

(c)

FIGURE 4: Immunofluorescence of the transplanted limbal graft showing growth of epithelial cells. (a) High magnification image of the graft showing stratified epithelium. (b) Epithelial cells stained with keratin 12 antibody (red), a marker for differentiated corneal epithelium. Nuclei are stained with DAPI. (c) Low magnification image of the graft stained with H\&E shows intensive inflammation.

The excimer laser has been in use for over two decades in refractive surgery. The Nidek EC-5000 laser system used in this study uses a $193 \mathrm{~nm}$ wavelength laser to efficiently remove corneal layers while limiting collateral thermal damage to the surrounding tissues [25]. The components of the corneal stroma, such as proteins, glycosaminoglycans, and nucleic acids, absorb energy of this wavelength and prevent any secondary mechanical shock-waves from transmitting through the eye, thus reducing any unwanted damage [26]. Our study is the first to use excimer to create an experimental model of limbal injury. It will be interesting to further investigate this limbal stem cell deficiency model with various degrees of limbal injury

Our in vivo results showed that the limbal extracellular matrix has a therapeutic effect on healing the epithelium and corneal surface. It was evident that as early as 7 days, the corneal surface damaged by laser ablation had healed better and appeared clearer with very little haze and neovascularization compared to the control nongrafted eyes. The grafts were covered by epithelium demonstrating a corneal phenotype. 


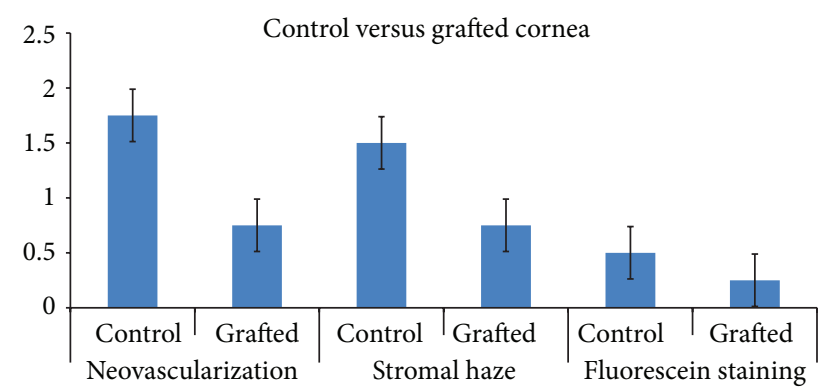

FIGURE 5: Statistical analysis of grafted and control cornea regarding degree of neovascularization, stromal haze and epithelialization defects. Fluorescein staining is used for quantifying epithelial defect. The differences between the control (laser only) and the grafted cornea were not statistically different. The following scoring system is used for quantification. Scoring: " 0 " is none (transparent, clear cornea), "1" is mild (minimal loss of transparency, mainly the epithelium is damaged), " 2 " is moderate (cloudy appearance, involves epithelium and part of the stroma), " 3 " is severe (opaque cornea), and " 4 " is total (opaque, involvement of entire thickness of the cornea).

At one week the grafts did not show any infiltration of fibroblasts into the transplanted graft. The keratocytes in the cornea are normally in a quiescent state which suggests that a longer time must be required to see any cell growth into the graft. It has been shown that any surgical incision or mechanical stress on the cornea that damages the tissue causes the keratocytes within $200-300 \mu \mathrm{m}$ of the cut to undergo apoptosis within a few hours after the surgery [27]. This creates a region of tissue devoid of cells surrounding the site of injury. A study on decellularized porcine corneal grafts observed a period of 4 weeks after grafting before infiltration of rabbit keratocytes [28]. Therefore, a longer study period is required to observe the migration of fibroblasts into the graft.

In summary, we have reported early in vivo data that a decellularized limbal graft can promote epithelial healing and restoration of the corneal epithelium. This opens the possibility of using decellularization grafts in corneal and limbal tissue engineering.

\section{Conflict of Interests}

The authors declare that they have no conflict of interests regarding the publication of this paper.

\section{References}

[1] M. Davanger and A. Evensen, "Role of the pericorneal papillary structure in renewal of corneal epithelium," Nature, vol. 229, no. 5286, pp. 560-561, 1971.

[2] A. Schermer, S. Galvin, and T.-T. Sun, "Differentiation-related expression of a major $64 \mathrm{~K}$ corneal keratin in vivo and in culture suggests limbal location of corneal epithelial stem cells," Journal of Cell Biology, vol. 103, no. 1, pp. 49-62, 1986.

[3] S. C. G. Tseng, "Concept and application of limbal stem cells," Eye, vol. 3, no. 2, pp. 141-157, 1989.

[4] R. M. Lavker, S. C. G. Tseng, and T.-T. Sun, "Corneal epithelial stem cells at the limbus: looking at some old problems from a new angle," Experimental Eye Research, vol. 78, no. 3, pp. 433446, 2004.

[5] J. J. Y. Chen and S. C. G. Tseng, "Corneal epithelial wound healing in partial limbal deficiency," Investigative Ophthalmology and Visual Science, vol. 31, no. 7, pp. 1301-1314, 1990.

[6] A. J. W. Huang and S. C. G. Tseng, "Corneal epithelial wound healing in the absence of limbal epithelium," Investigative Ophthalmology and Visual Science, vol. 32, no. 1, pp. 96-105, 1991.

[7] M. P. de Miguel, J. L. Alio, F. Arnalich-Montiel et al., "Cornea and ocular surface treatment," Current Stem Cell Research and Therapy, vol. 5, no. 2, pp. 195-204, 2010.

[8] B. Han, I. R. Schwab, T. K. Madsen, and R. R. Isseroff, "A fibrin-based bioengineered ocular surface with human corneal epithelial stem cells," Cornea, vol. 21, no. 5, pp. 505-510, 2002.

[9] M. Griffith, R. Osborne, R. Munger et al., "Functional human corneal equivalents constructed from cell lines," Science, vol. 286, no. 5447, pp. 2169-2172, 1999.

[10] D. J. Carlsson, F. Li, S. Shimmura, and M. Griffith, "Bioengineered corneas: how close are we?" Current Opinion in Ophthalmology, vol. 14, no. 4, pp. 192-197, 2003.

[11] K. Nishida, M. Yamato, Y. Hayashida et al., "Functional bioengineered corneal epithellial sheet grafts from corneal stem cells expanded ex vivo on a temperature-responsive cell culture surface," Transplantation, vol. 77, no. 3, pp. 379-385, 2004.

[12] J. Menzel-Severing, "Emerging techniques to treat limbal epithelial stem cell deficiency," Discovery Medicine, vol. 11, no. 56, pp. 57-64, 2011.

[13] M. Pauklin, T. A. Fuchsluger, H. Westekemper, K.-P. Steuhl, and D. Meller, "Midterm results of cultivated autologous and allogeneic limbal epithelial transplantation in limbal stem cell deficiency," Developments in Ophthalmology, vol. 45, pp. 57-70, 2010.

[14] C. Osei-Bempong, C. Henein, and S. Ahmad, "Culture conditions for primary human limbal epithelial cells," Regenerative Medicine, vol. 4, no. 3, pp. 461-470, 2009.

[15] F. Li, D. Carlsson, C. Lohmann et al., "Cellular and nerve regeneration within a biosynthetic extracellular matrix for corneal transplantation," Proceedings of the National Academy of Sciences of the United States of America, vol. 100, no. 26, pp. $15346-$ 15351, 2003.

[16] S. Shimmura, C. J. Doillon, M. Griffith et al., "Collagen-poly(Nisopropylacrylamide)-based membranes for corneal stroma scaffolds," Cornea, vol. 22, no. 7, pp. S81-S88, 2003.

[17] T. V. Chirila, "An overview of the development of artificial corneas with porous skirts and the use of PHEMA for such an application," Biomaterials, vol. 22, no. 24, pp. 3311-3317, 2001.

[18] R. J.-F. Tsai and R. Y.-N. Tsai, "Ex vivo expansion of corneal stem cells on amniotic membrane and their outcome," Eye and Contact Lens, vol. 36, no. 5, pp. 305-309, 2010.

[19] M. Grueterich, E. M. Espana, and S. C. G. Tseng, "Ex vivo expansion of limbal epithelial stem cells: amniotic membrane serving as a stem cell niche," Survey of Ophthalmology, vol. 48, no. 6, pp. 631-646, 2003.

[20] M. A. Shafiq, R. A. Gemeinhart, B. Y. J. T. Yue, and A. R. Djalilian, "Decellularized human cornea for reconstructing the corneal epithelium and anterior stroma," Tissue Engineering $C$, vol. 18, no. 5, pp. 340-348, 2012.

[21] A. R. Djalilian, A. Namavari, A. Ito et al., "Down-regulation of Notch signaling during corneal epithelial proliferation," Molecular Vision, vol. 14, pp. 1041-1049, 2008. 
[22] T. Kawakita, E. M. Espana, H. He, W. Li, C.-Y. Liu, and S. G. G. Tseng, "Intrastromal invasion by limbal epithelial cells is mediated by epithelial-mesenchymal transition activated by air exposure," American Journal of Pathology, vol. 167, no. 2, pp. 381393, 2005.

[23] S.-E. Ti, D. Anderson, A. Touhami, C. Kim, and S. C. G. Tseng, "Factors affecting outcome following transplantation of ex vivo expanded limbal epithelium on amniotic membrane for total limbal deficiency in rabbits," Investigative Ophthalmology and Visual Science, vol. 43, no. 8, pp. 2584-2592, 2002.

[24] F. L. Gimeno, V. Lavigne, S. Gatto, J. O. Croxatto, L. Correa, and J. E. Gallo, "Advances in corneal stem-cell transplantation in rabbits with severe ocular alkali burns," Journal of Cataract and Refractive Surgery, vol. 33, no. 11, pp. 1958-1965, 2007.

[25] T. Sakimoto, M. I. Rosenblatt, and D. T. Azar, "Laser eye surgery for refractive errors," The Lancet, vol. 367, no. 9520, pp. 14321447, 2006.

[26] E. E. Manche, J. D. Carr, W. W. Haw, and P. S. Hersh, "Excimer laser refractive surgery," Western Journal of Medicine, vol. 169, no. 1, pp. 30-38, 1998.

[27] M. E. Fini, "Keratocyte and fibroblast phenotypes in the repairing cornea," Progress in Retinal and Eye Research, vol. 18, no. 4, pp. 529-551, 1999.

[28] X.-C. Lin, Y.-N. Hui, Y.-S. Wang, H. Meng, Y.-J. Zhang, and Y. Jin, "Lamellar keratoplasty with a graft of lyophilized acellular porcine corneal stroma in the rabbit," Veterinary Ophthalmology, vol. 11, no. 2, pp. 61-66, 2008. 


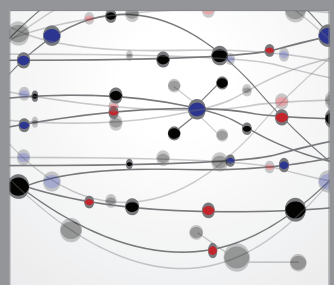

The Scientific World Journal
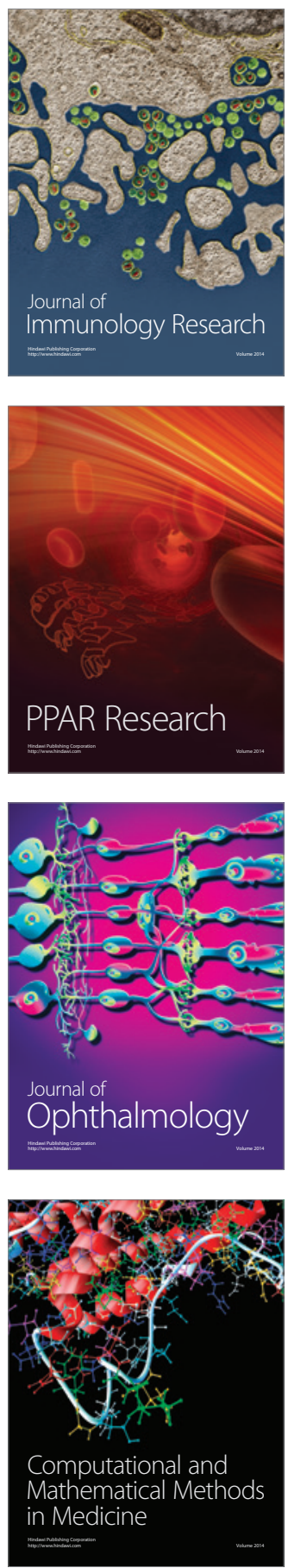

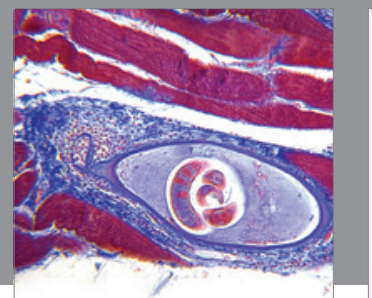

Gastroenterology

Research and Practice
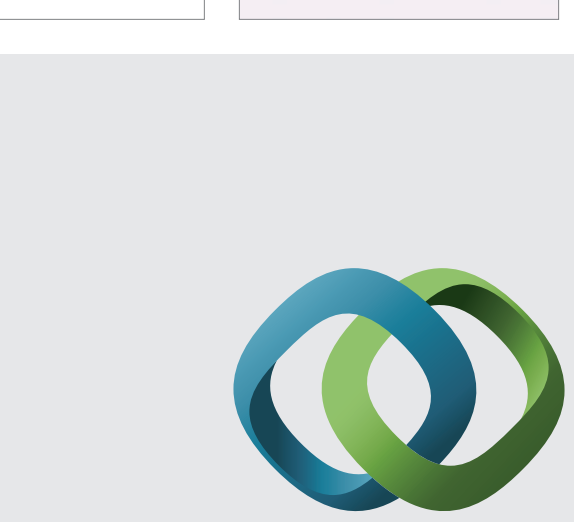

\section{Hindawi}

Submit your manuscripts at

http://www.hindawi.com
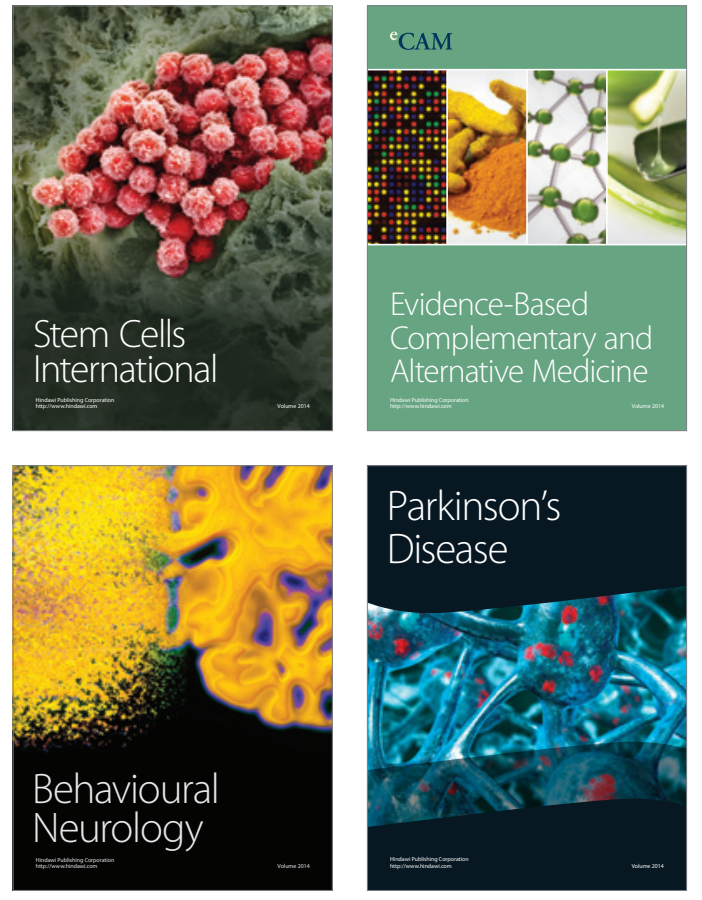
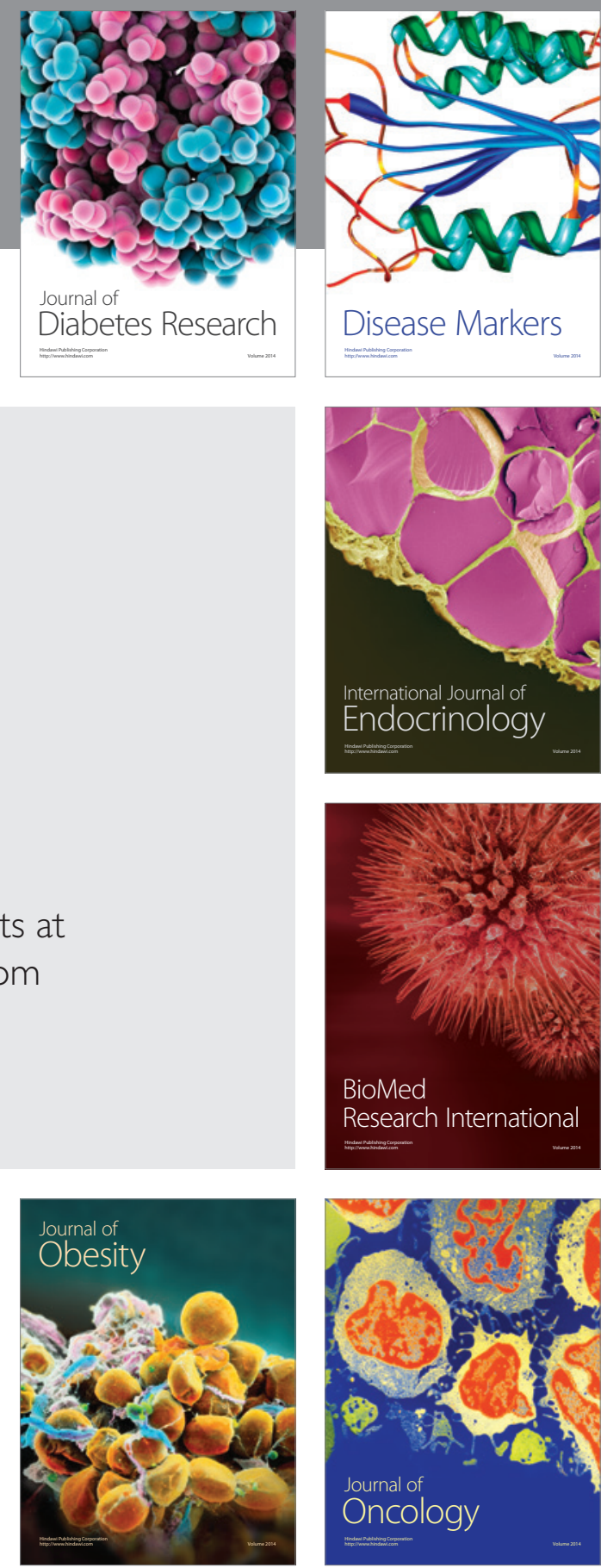

Disease Markers
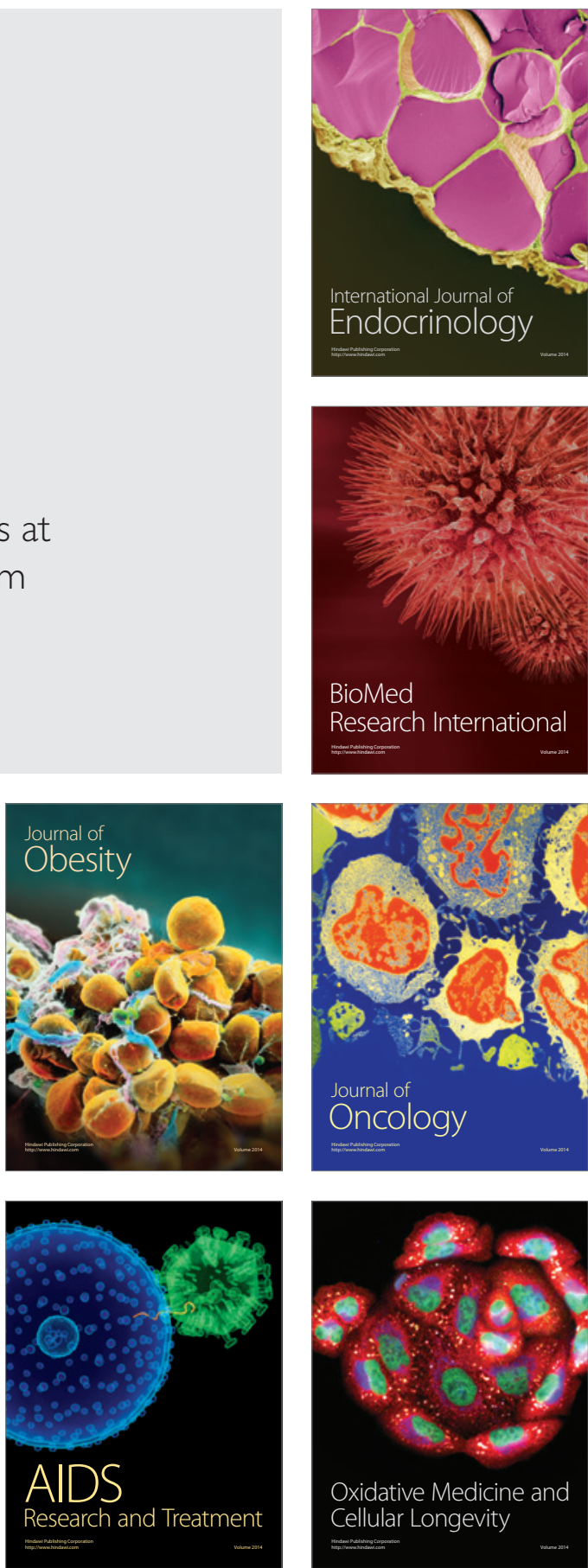